\title{
Primary and secondary overacting inferior oblique muscles: an ultrastructural study
}

\author{
E. MEYER, R. M. LUDATSCHER, AND S. ZONIS \\ From the Departments of Ophthalmology and Pathology, Rambam Medical Center and Faculty of Medicine, \\ Haifa, Israel
}

SUMMARY Biopsy material from primary and secondary overacting inferior oblique muscles were examined by light and electron microscopy. Most muscle fibres were in different stages of atrophy. A high variation of alterations was encountered in all muscles. The most striking abnormalities were huge accumulations of mitochondria and muscle vacuolisation related mainly to the enlargement of the tubules of sarcoplasmic reticulum. The mitochondrial aggregates and vacuolisation occupied more muscle surface in the inferior oblique muscles of primary overaction than those of secondary overaction.

The disturbances of ocular motility often include overaction of the inferior oblique muscle. ${ }^{1-3}$ From the clinical point of view there are two types of overaction. ${ }^{3}$ The primary type usually deals with bilateral overacting inferior oblique muscles and is of unknown cause, whereas the secondary type is usually unilateral and is related to the palsy of the superior oblique. ${ }^{3}$ The problem of providing a cause for the primary overacting inferior oblique muscle is amply emphasised by Spencer and McNeer. ${ }^{3}$ The authors, in a complete ultrastructural study, conclude that primary and secondary overacting inferior oblique muscles contain similar morphological alterations of the muscle fibres. Their findings lead to the possibility that the primary overacting inferior oblique is the result of a bilateral paresis of the superior oblique muscle.

The aim of this study is to enlarge our knowledge of the ultrastructural alterations encountered in overacting inferior oblique muscles and to define the structural differences between primary and secondary overaction as seen in our series of muscles.

\section{Material and methods}

This study deals with 10 biopsies of overacting inferior oblique muscles from eight patients ranging between 6 and 18 years of age. Of these, six had primary overacting inferior obliques associated in all cases

Correspondence to Dr Ewy Meyer, Department of Ophthalmology, Rambam Medical Center, Haifa, Israel. with esotropia, and two had secondary overacting inferior oblique muscles due to paralysis of the superior oblique muscle with a positive Bielchowsky head tilt test. All cases except one had strabismus at birth onset. A non-overacting inferior oblique muscle was obtained from a patient undergoing enucleation for intraocular disease as a control specimen.

The myectomy procedure as described in von Noorden's book ${ }^{4}$ was used to weaken the overacting inferior oblique muscle surgically. All the biopsies were taken by the same surgeon (E.M.). On removal the specimens were immediately cut into small pieces and fixed with ice cold $3 \%$ glutaraldehyde in cacodylate buffer $(\mathrm{pH} \mathrm{7 \cdot 3)}$ for one hour, postfixed with $2 \%$ osmium tetroxide, dehydrated in alcohols, and embedded in Epon. A section $1 \mu \mathrm{m}$ thick was stained with $1 \%$ toluidine blue and examined with a light microscope. Thin sections were stained with uranyl acetate and lead citrate and examined with a Zeiss $9 \mathrm{~S}$ electron microscope.

\section{Results}

PRIMARY OVERACTING INFERIOR OBLIQUE MUSCLES

In this group the muscle fibres showed large variations in size, the diameter varying between 10 and $20 \mu \mathrm{m}$ (Fig. 1). The muscle fibres showed the characteristic features of human extraocular muscles..$^{4-7}$ Usually round bodies of fine granular material appeared subsarcolemmally or adjacent to the nucleus. In two muscles subsarcolemmal inclusions composed of 


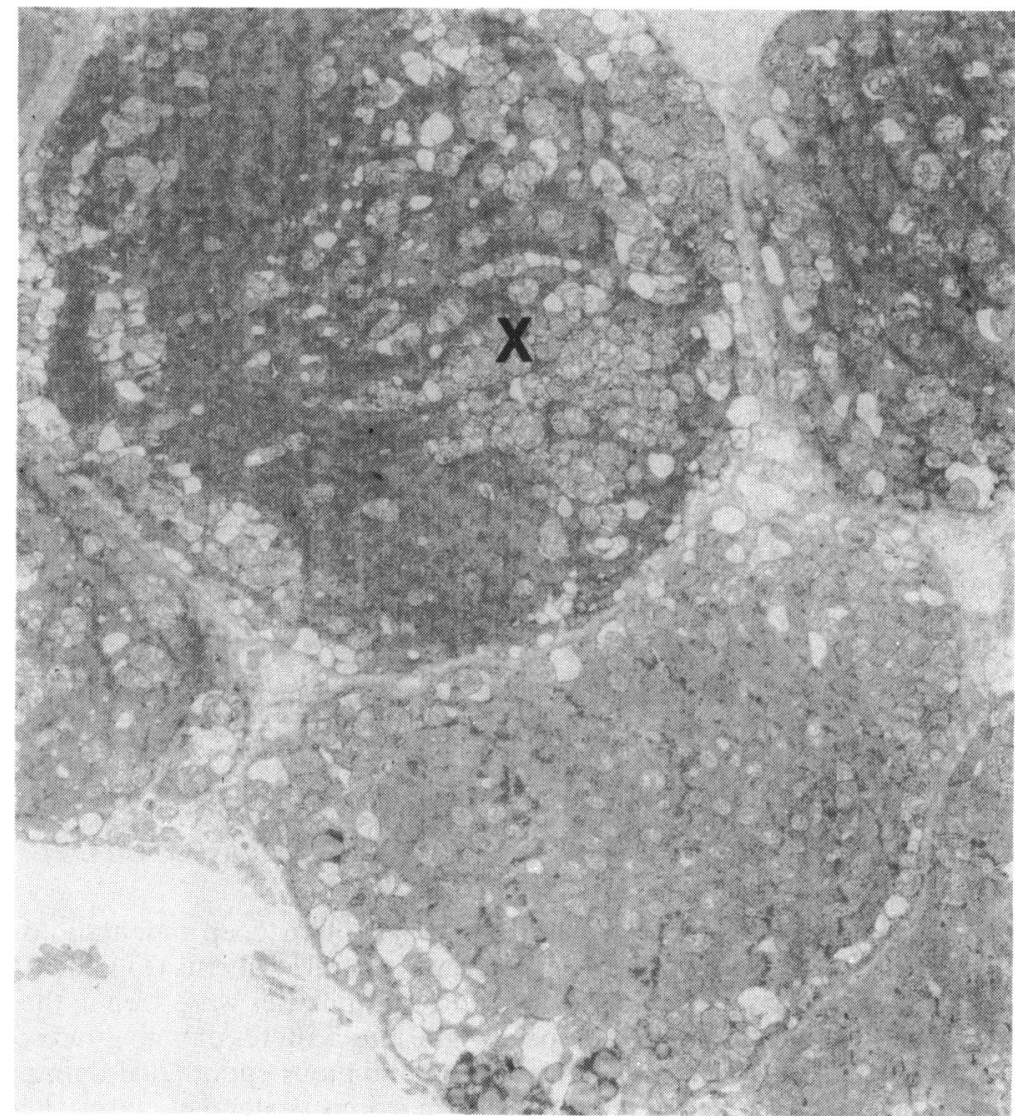

Fig. 1 Primaryoveraction. The muscle fibres show variation in size and shape, the largest cell measuring $18 \mu \mathrm{m}$. Large aggregates of mitochondria (X) and empty vacuoles occupy a sizeable part of the muscle fibre. $(\times 3800)$.

parallel and elongated thin fibres, known as Hirano bodies, were encountered. ${ }^{58}$

The most striking abnormalities consisted of large aggregates of mitochondria which occupied a great part of the muscle cell surface (Fig. 1). The mitochondrial clusters were located at random with no topographical predilection. The mitochondria also showed variation in size and configuration. Disruption and loss of mitochondrial cristae creating aspects of empty mitochondria were often visible (Fig. 2).

Muscle cells containing aggregates of mitochondria usually showed numerous empty vacuoles of varied size and shape (Figs. 1, 2). In photographs of higher magnification it was apparent that the empty vacuoles represented enlarged tubules of sarcoplasmic reticulum and only a few were related to empty mitochondria (Fig. 2).

In some muscle fibres the vacuolisation and the increased number of mitochondria were less prominent, and these cells were differently altered. The alterations included disorganisation of sarcomeres, disruption of myofilaments, and accumula- tion of myofilaments into smudgy masses (Fig. 3). Sometimes the Z-band was distorted or disrupted (Fig. 3). Occasionally nemaline (rod) bodies and contraction bands were noticed. In some muscles groups of contracted sarcomeres appeared adjacent to stretched sarcomeres.

The endomysial collagen tissue was moderately increased and contained myelinated and unmyelinated axons.

\section{SECONDARY OVERACTING INFERIOR OBLIQUE} MUSCLE

In this group the muscle fibres were similar in size and shape to those of the first group. Contraction bands and asymmetrically contracted sarcomeres, disruption of the sarcomere pattern, and alterations of the $\mathrm{Z}$ band were encountered (Fig. 4). In comparison with the first group of muscles it became apparent that the amount of endomysial collagen fibrils was markedly increased and that the amount of aggregated mitochondria and of empty vacuoles was less striking (Fig. 5). On the other hand, though the clusters of mitochondria were not so numerous, the 
Fig. 2 Primaryoveraction. The sarcomere pattern is disrupted, and the mitochondria contain numerous cristae. Loss of cristae appears in several mitochondria (X). Groups of empty, single membrane enclosed vacuoles are evident. $(\times 15724)$.

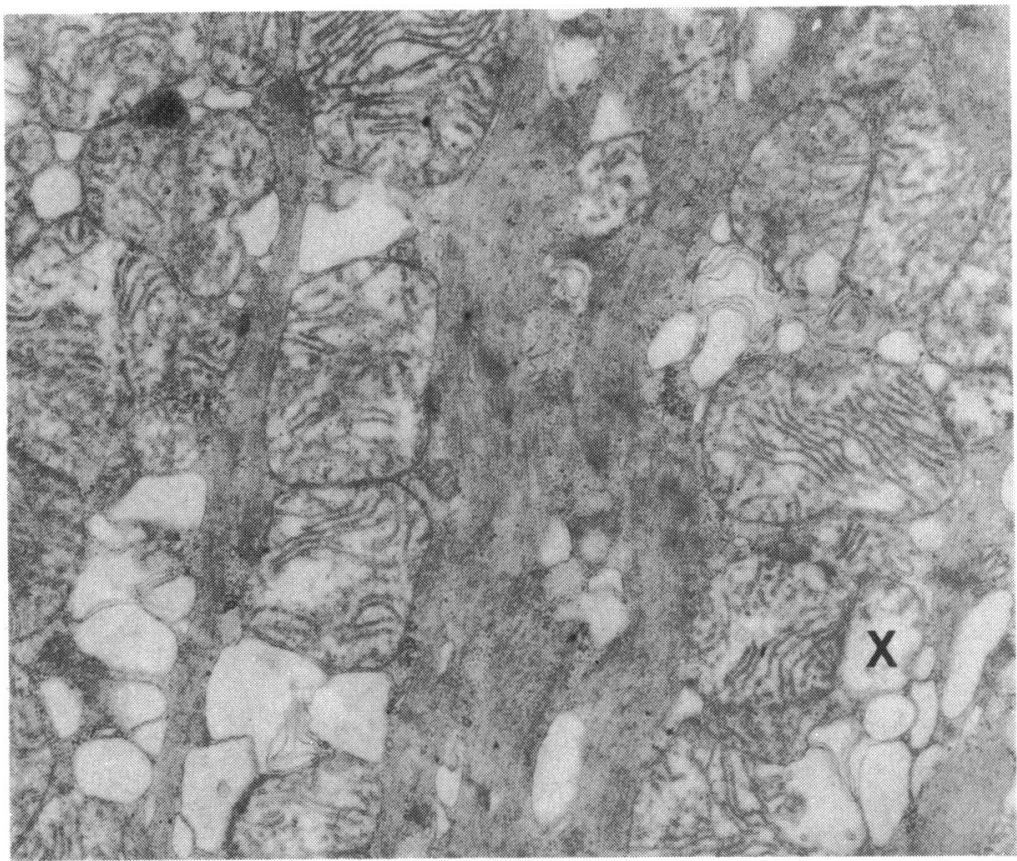

abnormalities in mitochondrial size and cristae were common.

Nuclei. In both groups the majority of muscle fibres had the nuclei in a normal subsarcolemmal location, but migration of nuclei to the centre of the cell was encountered (Fig. 5). The nuclei had an irregular convoluted envelope, and often deep indentations divided the nucleus into several segments (Fig. 5).

Capillaries. Numerous capillaries were seen in the endomysial space of the muscle fibres of both groups. The capillaries had a continuous endothelial lining, and endothelial projections extended into the
Fig. 3 Primaryoveraction. The $Z$ band is disrupted $(\mathrm{Z})$ and the degenerated myofilaments are accumulating into focal masses (S). $(\times 15724)$.

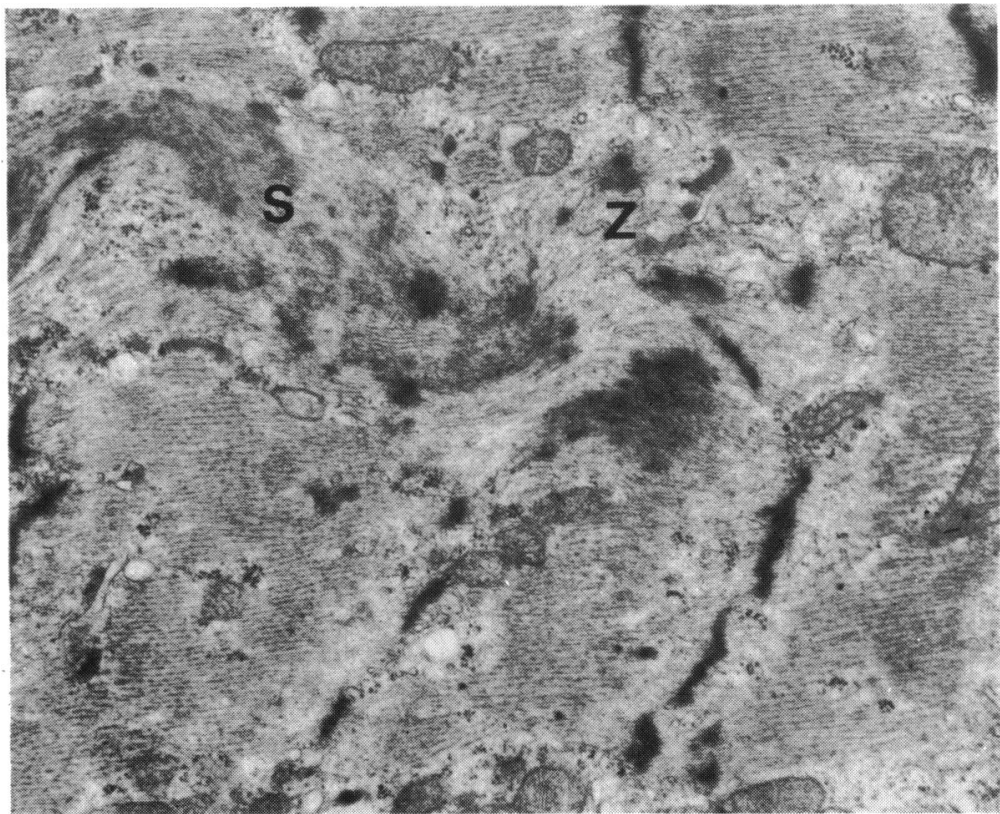




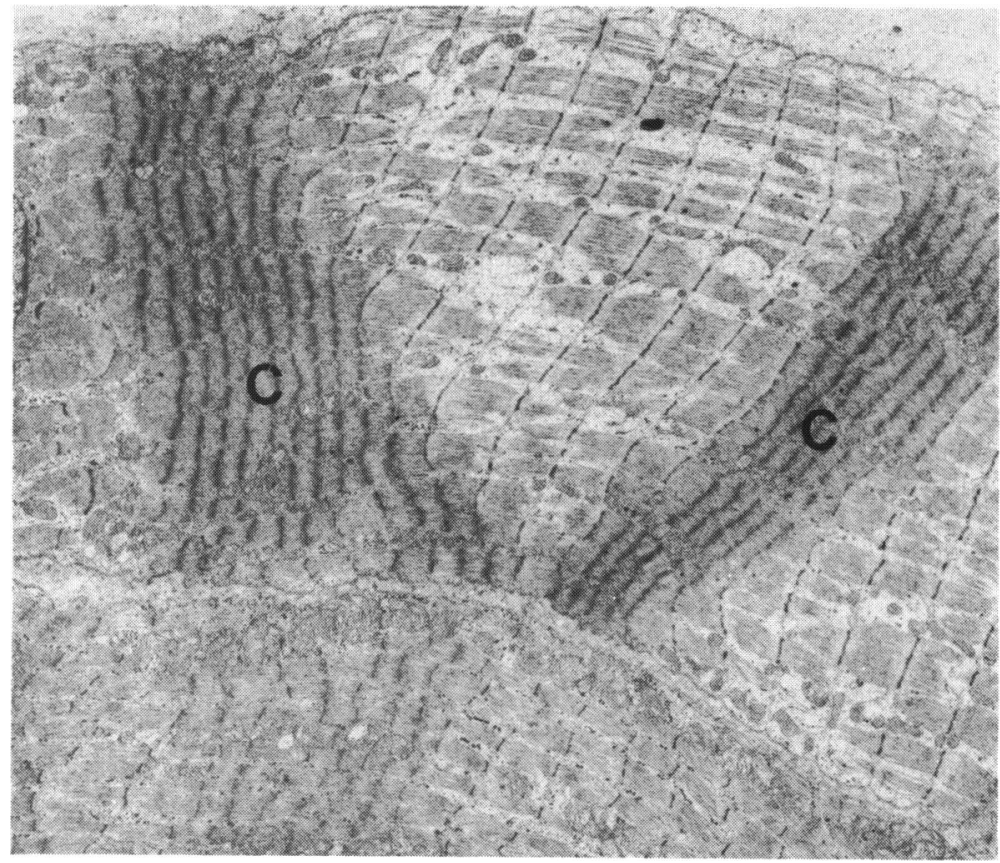

Fig. 4 Secondary overaction. The lower muscle fibre shows 2 contraction bands $(\mathrm{C})$ on both sides of an area with normally stretched sarcomeres. The upper muscle fibre shows asymmetrically contracted sarcomeres. $(\times 3442)$.

vascular lumen. A wide basement membrane 160-220 $\mathrm{nm}$ in diameter delineated the outer side of the capillaries.

Control inferior oblique muscle. No marked vacuolisation or large aggregates of mitochondria were noticed.

\section{Discussion}

The rationale for a comparative study of the alterations of primary and secondary overacting inferior oblique muscle was to try to find criteria of differentiation for each type.

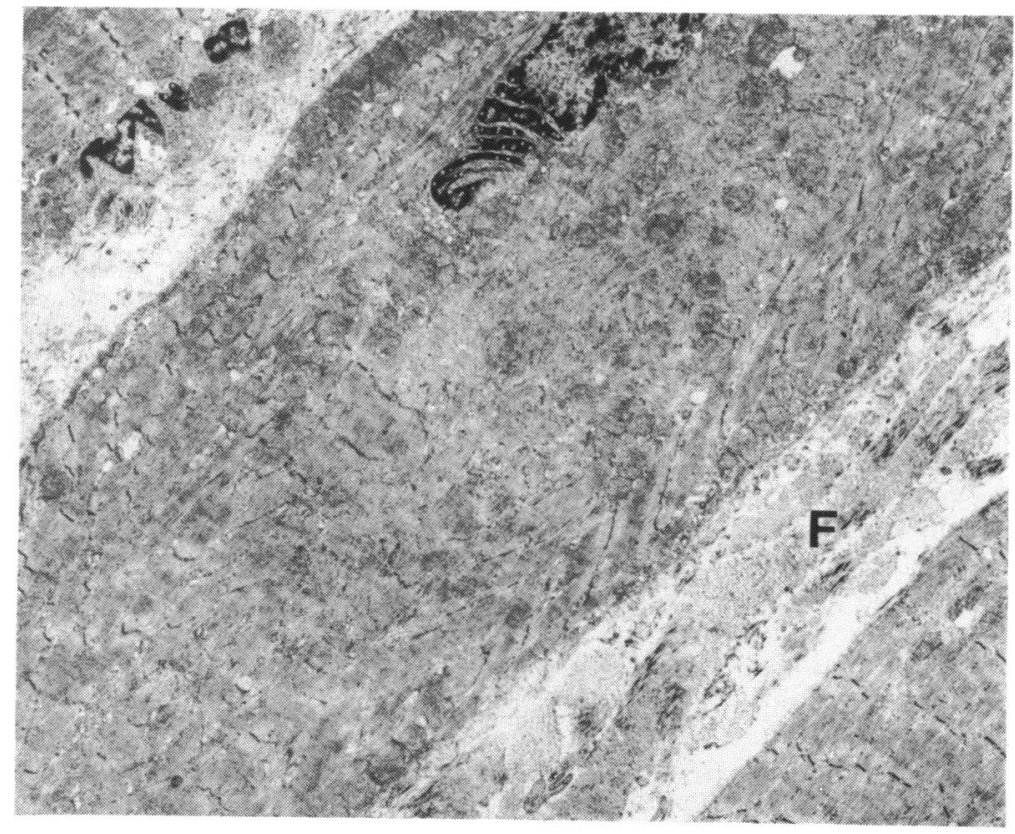

Fig. 5 Secondaryoveraction. The muscle fibres show disrupted sarcomeres and irregularities of the $Z$ band. The nucleus on the top of the picture is displaced towards the centre of the muscle fibre and appears segmented into several portions. The endomysial space between muscle fibres is occupied by a large amount of collagen fibrils (F). (×3292). 
A large variation of alterations in muscle cell structure was encountered in the present series of overacting inferior oblique muscles. The most striking abnormalities were huge accumulations of mitochondria and muscle vacuolisation related mainly to enlargement of tubules of sarcoplasmic reticulum. These changes are frequently noticed in other series of overacting inferior oblique muscles. ${ }^{3}$ However, in agreement with others ${ }^{3}$ we should point out the absolute non-specificity of the lesions. Mitochondrial aggregates and vacuoles were described in myasthenia gravis, ${ }^{9}$ in Apert's syndrome, ${ }^{8}$ in Duane's syndrome, ${ }^{10}$ and in varied myopathic states. ${ }^{11}$ Mitochondrial abnormalities are believed to occur also in normal extraocular muscle. ${ }^{5}$ It is well known that mitochondrial alterations are common in varied pathological states of extraocular muscles and of skeletal muscles as well.

Considering the present results and those from the literature, we believe that a certain leading point could be reached by analysing the amount of mitochondrial aggregates and of vacuoles occupying the muscle cell. In our series of primary overacting muscles, except for one case, the mitochondrial aggregates and the empty vacuoles occupy about half of the muscle cell surface, whereas in secondary overaction mitochondrial aggregates and vacuolisation are less prominent.

Most of the muscle fibres are in different stages of atrophy. However, the deeply invaginated nuclei and the contraction bands would suggest that a state of hypertrophy had taken place beforehand. Contraction bands and contracted sarcomeres were encountered in many muscles of both series, and these appeared always in muscle cells containing few mitochondria. Contraction bands are non-specific changes and were thought in the past to be the result of artefactual fixation. ${ }^{1213}$ However, in a recent experimental study it was shown that asymmetrically contracted sarcomes are not artefactual, and they develop in tetanised muscle. ${ }^{14}$ In our material contraction bands and stretched sarcomeres were seen to alternate in the same cell, therefore excluding the possibility of artefactual changes. The possibility that the supercontracted myofibrils could represent a state of contracture or failure of relaxation owing to a relative deficiency of adenosine triphosphate (ATP), as postulated in cases with Duchenne muscular dystrophy, ${ }^{12}$ may well apply to the muscle fibres which contained few mitochondria and therefore were deficient in ATP.

Mukuno et al. ${ }^{15}$ suggest that myopathic lesions resulting from abnormal mitochondrial activity could cause overaction of the inferior oblique muscle in patients with various types of strabismus.

With regard to the marked vacuolisation encountered in our muscles of primary overaction, these changes are comparable to the dilated sarcoplasmic tubules of vacuolar myopathy described in patients with hypokalemic periodic paralysis. ${ }^{16}$

The structural findings in primary overacting muscles raise the possibility of myopathic changes involving the extraocular muscles and affecting also the superior oblique muscle with paresis of this muscle. The overacting inferior oblique muscle possibly undergoes hypertrophy in the initial stage and becomes atrophic in a later stage.

Our thanks are due to Mrs $\mathbf{Z}$. Weinberg for excellent technical assistance.

\section{References}

1 Ghi S. Study of the A-V patterns in horizontal strabismus. Nippon Ganka Gakkai Zasshi 1969; 73: 886-97.

2 Parks MM. The overacting inferior oblique muscle. Am J Ophthalmol 1974; 77: 787-96.

3 Spencer RF, McNeer KW. Structural alterations in overacting inferior oblique muscles. Arch Ophthalmol 1980; 98: 128-133.

4 von Noorden GK. Binocular vision and ocular motility. St Louis: Mosby, 1980.

$5 \mathrm{McNeer} \mathrm{KW}$. Histopathology of extraocular muscles in strabismus. In: Helveston EM, ed. Symposium on strabismus. St Louis: Mosby, 1978: 80-90.

6 Muhlendyck H, Ali SS. Histological and ultrastructural studies on the ringbands in human extraocular muscles. Albrecht von Graefes Arch Klin Ophthalmol 1978; 208: 179-91.

7 Berard-Badier M, Pellissier JF, Toga M, Monillac N, Berard PV. Ultrastructural studies of extraocular muscles in ocular motility disorders. Albrecht von Graefes Arch Klin Ophthalmol 1978; 208: 193-205.

8 Margolis S, Pachter BR, Breinin GM. Structural alterations of extraocular muscle associated with Apert's syndrome. $\mathrm{Br} J$ Ophthalmol 1977; 61: 683-9.

9 Sakimoto T. Electron microscopic studies on human extraocular muscles: III. Fine structural finding in extraocular muscles with descending ocular myopathy. Nippon Ganka Gakkai Zasshi 1971; 75: 748-64.

10 Ludatscher RM, Meyer E, Zonis S. Electron microscopic study of Duane's syndrome, endocrine ophthalmopathy and other myopathies with strabismus. Ophthalmic Paediatr Genet 1982; 1: 211-8.

11 Adachi M, Torii J, Vold BW, Briet P, Wolintz A, Schneck L. Electron microscopic and enzyme histochemical studies of cerebellum, ocular and skeletal muscles in chronic progressive ophthalmoplegia with cerebellar ataxia. Acta Neuropathol 1973; 23: 300-8.

12 Mastaglia FL, Papadimitriou JM, Kakulas BA. Regeneration of muscle in Duchenne muscular dystrophy: an electron microscopic study. J Neurol Sci 1970; 11: 425-44.

13 Van Noorden S, Olsen EGJ, Pearse AGE. Hypertrophic obstructive cardiomyopathy, a histological and ultrastructural study of biopsy material. Cardiovasc Res 1971; 5: 118-31.

14 Bergman RA. Ultrastructural configuration of sarcomeres in passive and contracted frog sartorius muscle. Am J Anat 1983; 166: 209-22.

15 Mukuno K, Ishikawa S, Togo T, Minei Y. Histopathological study on the overacted inferior oblique muscles with special reference to central core within the muscle fibres. Jpn J Ophthalmol 1976; 20: 166-76.

16 Howes EL, Price HM, Pearson CM, Blumberg JM. Hypokalemic periodic paralysis. Neurology 1966; 16: 242-56. 